\title{
Control of an Adaptive Light Shelf Using Multi-Objective Optimization
}

\author{
Benny Raphael $^{a}$ \\ ${ }^{a}$ Civil Engineering Department, IIT Madras, Chennai, India \\ E-mail: benny@iitm.ac.in
}

\begin{abstract}
-
Users are increasingly expecting intelligent behavior from automated building systems. However, incorporating and integrating user preferences in an automatic control algorithm is a complex task. In most commercial systems, control is done through local search techniques such as Proportional Integral Derivative (PID) control. Multiple criteria cannot be accommodated in such techniques. The objective of this paper is to evaluate the use of multi-criteria optimization in the control of a lighting and shading device, an adaptive light shelf. This study is done in two stages. In the first stage, the performance of the light shelf is evaluated using simulations. EnergyPus and Radiance are used to simulate thermal load and daylighting. These are used to determine the optimal control actions using a multi-objective optimization algorithm. The energy savings for electrical lights is compared with a traditional control strategy. In the second stage, a prototype is constructed to evaluate the actual performance. Results from simulation show that significant energy savings can be achieved through multi-objective optimization control strategy.
\end{abstract}

Keywords -

Building automation, Control, lightshelf, multiobjective optimization, energy efficiency

\section{Introduction}

Traditionally control is treated as a single objective optimization problem and the best solution according to a selected criterion is chosen as the control action. However, building systems are getting increasingly complex and there is a need to accommodate multiple criteria for the best performance. For example, an external window blind might be partially opened or closed in order to satisfy requirements such as eliminate glare, provide adequate daylight, reduce cooling load and accommodate user preferences.

In multi-criteria optimization, several objectives are considered simultaneously and usually these conflict with each other. For example, minimizing the lighting energy might require opening the window blinds which would increase the radiant heat entering into the building. Therefore, in multi-objective optimization the emphasis is on identifying solutions that achieve the best trade-offs among multiple objectives. An early application of this concept can be found in Radford and Gero [1] where they applied dynamic programming in the multi-criteria design optimization with four performance criteria namely, thermal load, daylight availability, construction cost and usable area..

A popular approach to multi-objective optimization is the generation of a Pareto Front. In this approach, a population of solutions known as the Pareto set is generated in which all the solutions are Pareto optimal or non-dominated. By definition, there is no solution better than a Pareto optimal solution with respect to all the criteria simultaneously. Several applications of this concept are found in the literature (eg. [2,3]). Some of these techniques have already been used in the optimization of building systems. Caldas [4] studied the trade-off between the initial cost of a building and the energy performance of the building using a multiobjective optimization approach.

While the Pareto approach is useful for design tasks in helping engineers make decisions, it does not support the selection of a single best solution. Automatic selection of solutions is needed in tasks such as control where decisions have to be taken several times a minute or second. The issue of selecting a single solution from the Pareto set has been largely ignored by previous researchers. This paper presents a new algorithm called RR-PARETO3 for selecting the best solution that makes reasonable trade-offs among conflicting objectives.

\section{RR-PARETO3 Algorithm}

RR-PARETO3 algorithm is the third generation of the multi-criteria decision making algorithm presented in [5]. In this algorithm, the solution with the best tradeoffs among all the objectives is chosen using two pieces of information: 
- Order of the objectives according to their importance

- The sensitivity of each objective

The sensitivity of an objective specifies what level of increase in its value is acceptable to the user. For example, consider the objective of minimizing the power consumption. If increasing the power consumption above $5 \%$ is not acceptable to the user, the sensitivity of this objective is defined as $5 \%$. In this case, the algorithm attempts to select solutions that are within $5 \%$ of the best solution. In the following discussion, the term solution point or point is used to denote a set of values of all the objectives as well as the decision variables (optimization variables).

The RR-PARETO3 algorithm works by iteratively eliminating the worst points according to maximum number of criteria. This filtering is done in two stages. In the first stage, the solution point with the best value for the current objective is chosen from among all the points. All the points that lie outside the sensitivity band of the chosen point are eliminated from the set. If the sensitivity is not specified for any objective, no filtering is done for this objective and all the solutions are retained. At the end of Stage 1, one or more points might remain in the solution set. If a unique solution is not identified, Stage 2 filtering is performed.

In Stage 2 filtering, the hypercube containing all the remaining solutions is trimmed. This is done by dividing the hypercube volume into half by bisecting each objective axis one by one according to their order of importance. Let $\mathrm{ymin}_{\mathrm{i}}$ and $\mathrm{ymax}_{\mathrm{i}}$ be the minimum and maximum values of the $i$-th objective among all the solutions in the current set. The threshold is computed as $\left(\mathrm{ymin}_{\mathrm{i}}+\mathrm{ymax}_{\mathrm{i}}\right) / 2$. In the minimization problem, the region containing values greater than this threshold is considered as the bad half with respect to this objective. Worst solutions are eliminated using the algorithm described below:

- $\quad$ Stage 2.1: Points that lie within the bad half of most objectives are eliminated, taking combinations of $\mathrm{k}$ most important objectives at a time (repeated for $\mathrm{k}=\mathrm{N}$ to 2 )

- Stage 2.2: Points that lie in the bad half of individual objectives are eliminated according to the order of importance of objectives

- Stage 2.3: Iteratively remove the worst point according to each objective based on the order of importance

The process of bisection of hypercube helps to remove visibly obvious bad solutions. When there are still many points left, each objective is given a chance to remove the worst candidate in Stage 2.2. The most important objective is given the first chance. Stages 2.1 and 2.2 use values of objective function for elimination, while Stage 2.3 uses ranking of solutions. The process stops when a single solution remains in the set or all the remaining solutions have the same values for all the objective functions. By repeating Stage 2.2 for each objective, each criterion is given an opportunity to eliminate bad solutions and the final selection is a tradeoff among all the objectives. It is emphasized that the process does not favour the best solution according to any objective. For example, if the best solution according to the first objective lies within the bad half of the second objective, this solution is eliminated. Since the process is driven by the order of importance of objectives, the users' preferences in the selection process are also respected.

The algorithm can be used to filter a set of solution points that are generated by any optimization algorithm or even pure random sampling procedures.

\section{Application to lightshelf control}

A lightshelf is a horizontal or inclined projection with a high reflectivity meant to increase the depth of daylight penetration into a room. It operates by reflecting sunlight off to the ceiling from where it is further reflected to the work plane. Several studies have been conducted on the effectiveness of light shelves and other daylighting features. For example, Aghemo et al. [6] present a case study for the comparison of lighting performances of different traditional shading devices.

If the light shelves are static and horizontal, they are able to increase daylight penetration only when the sun is at a low angle. A proposal to making the light shelf rotatable is presented in [7]. In this case, the angle can be adjusted to the position of the sun to produce deeper daylight penetration (Figure 1).

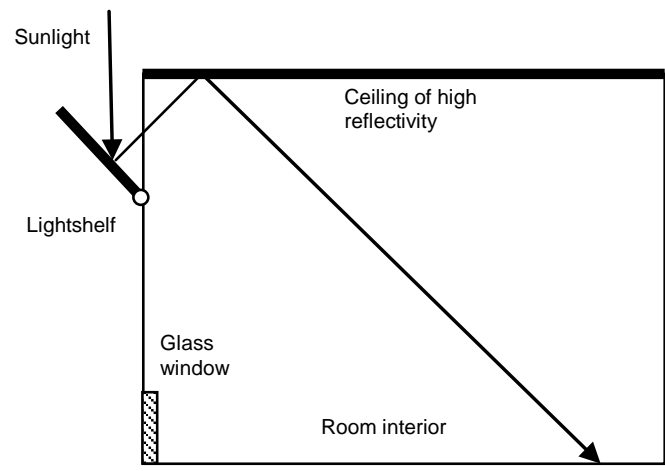

Figure 1. By adapting the angle of the light shelf to the position of the sun, deeper penetration of daylight is achieved 
The control of the light shelf is a complex task. When the external light shelf is rotated in the clockwise direction, initially there is an increase in daylight penetration and later at greater angles, daylight decreases depending on the angle of the sun. There is an optimal position of the light shelf at which adequate shading is provided near the window and maximum daylight is produced in the interior. In addition, the amount of heat reflected by the light shelf into the interior of the building as well as the amount of heat blocked by it have to be computed in order to evaluate its performance with respect to energy required for cooling the buildings. This makes it a multi-objective control task.

\subsection{Choosing values of algorithm Parameters}

The only algorithm parameters that need to be specified in RR-PARETO3 are the order of objectives and the sensitivity of objectives. The order of objectives depend on the priorities of the user. In this application lighting is given higher priority, because the primary objective of using a light shelf is to improve the lighting level. The second parameter, sensitivity, depends on how much decrease in performance is acceptable to the user. In the case of light shelf, the user has to decide on what percentage of increase in cooling load is acceptable. This is a subjective decision. If the user does not have any preference, the parameter can be omitted allowing RR-PARETO3 to apply the default filtering algorithm.

\subsection{Case study}

In order to evaluate the control strategy, a hypothetical office building of plan area 8.0 x 6.0 meter is considered here. The dimensions are shown in Figure 2. A rotatable light shelf of width $1.0 \mathrm{~m}$. is present on the west facade at a height of $2.0 \mathrm{~m}$. Dimmable lights of 56 Watts are installed along a grid of spacing $2 \mathrm{~m}$ in both directions. Other data related to the case study are given in Table 1.

Table 1 Case study data

\begin{tabular}{cc}
\hline Parameter & Value \\
\hline Light shelf reflectivity & $90 \%$ \\
Visible Transmittance of glass & $35 \%$ \\
U value of glass & 2.2 \\
Shading coefficient of glass & 0.35 \\
Weather Data & Chennai \\
& (India) \\
\hline
\end{tabular}

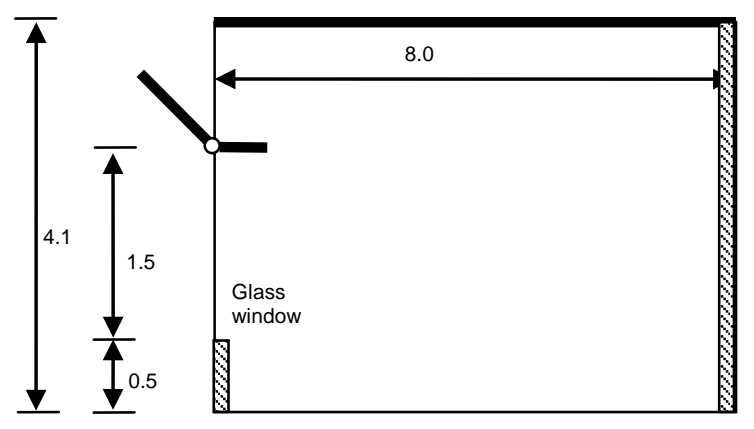

Figure 2. Case study of an office building

The daylight available in the room is calculated using lighting simulation software Radiance [8]. The thermal load is computed using EnergyPlus Version 8.1 [9]. In order to correctly calculate the effects of reflections from the light shelf, the "Solar Distribution" parameter in EnergyPlus is set to "Full Interior and Exterior With Reflections". Lighting power for the dimmable lamps was calculated from the illuminance values obtained through Radiance by assuming a linear dimming curve such that the power varies between $10 \%$ and $100 \%$ for minimum and maximum brightness of the lamp. In order to prevent glare, it is assumed that window blinds are completely closed if the maximum illuminance exceeds 2000 lux at a distance of 2 meters from the window. Hourly simulations are done for a typical summer day for different angles of the light shelf in order to determine the optimal position with respect to lighting and cooling energy criteria. As an illustration, the lighting and cooling loads for various angles of the light shelf are shown for 3 pm (Figures 3,4). The angle 0 corresponds to a horizontal light shelf and the angle 90 degrees corresponds to vertically upward position. The power does not increase or decrease monotonically because of the effects of local reflections as well as discretization and other errors in the simulations. It is clear that small random variations in the load should not be allowed to influence the optimal position. This justifies the use of the sensitivity parameter in the RRPARETO3 algorithm. Also it is noted that the nonmonotonicity of the objective function and the small random variations create problems for conventional local search algorithms. For example, a small step might seem to increase the value of the objective function and the algorithm might terminate assuming that a minimum is reached, while actually it might be a local effect due to errors. If such small random variations are ignored, a general increasing trend in lighting power is observed above 30 degrees. At the same time, there is a general decreasing trend in the solar thermal load. 


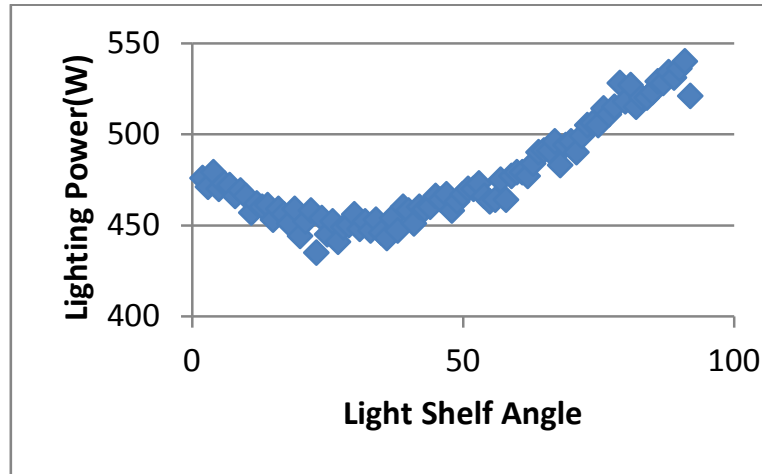

Figure 3. Variation of lighting power with light shelf angle at $3 \mathrm{pm}$

After computing the lighting power and solar thermal load for all the angles of the light shelf from $0^{\circ}$ to $90^{\circ}$, the compromise solution was obtained using RRPARETO3 filtering. Three scenarios were considered. In the first scenario, lighting criterion was given higher priority over solar thermal load. Both objectives were given sensitivity values of $5 \%$. In the second scenario, the solar thermal load criterion was given higher priority while keeping the sensitivity values unchanged. In the third scenario, the lighting criterion was assigned higher priority, while the sensitivity parameters were increased to $10 \%$. The results are presented in Table 2 .

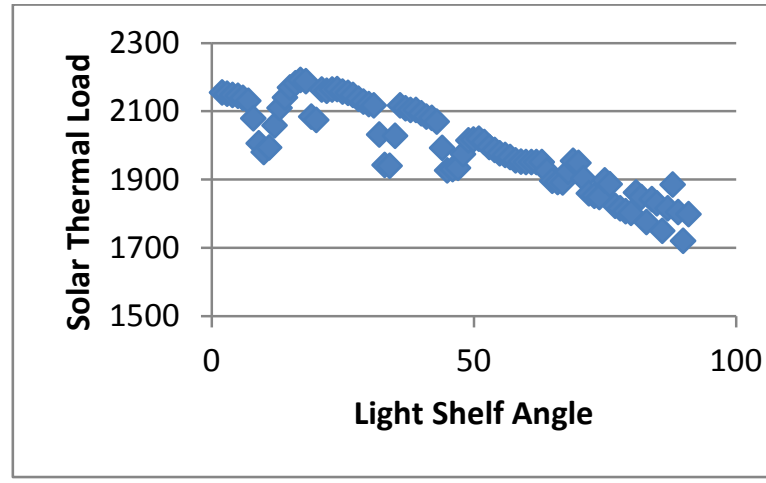

Figure 4. Variation of Solar Thermal Load with light shelf angle at $3 \mathrm{pm}$

In the first scenario, the compromise solution was the light shelf angle of 32 degrees which corresponds to lighting load of $453 \mathrm{~W}$ and solar thermal load of 1940 W. In the second scenario, the light shelf was completely closed (90 degree angle) which results in lighting load of $540 \mathrm{~W}$ and solar thermal load of 1799
W. In the third scenario, greater increases in lighting and solar thermal load of up to $10 \%$ were acceptable. With this setting, an optimal angle of 44 degrees was obtained.

Table 2 Compromise solutions at $3 \mathrm{pm}$

\begin{tabular}{cccc}
\hline Scenario & $\begin{array}{c}\text { Angle } \\
\text { (degree) }\end{array}$ & $\begin{array}{c}\text { Lighting } \\
\text { Power } \\
(\mathrm{W})\end{array}$ & $\begin{array}{c}\text { Solar } \\
\text { Thermal } \\
\text { load } \\
\text { (W) }\end{array}$ \\
\hline 1 & 32 & 453 & 1940 \\
2 & 90 & 540 & 1799 \\
3 & 44 & 464 & 1930
\end{tabular}

A single objective optimization control strategy in which the lighting power is minimized would result in an angle of 21 degrees with lighting power of $435 \mathrm{~W}$ and Solar Thermal load of $2164 \mathrm{~W}$. This solution has $11.5 \%$ higher solar thermal load while the decrease in lighting power is only 4\% compared to Solution 1. Therefore the solution identified by RRPARETO3 algorithm for scenario 1 represents a good trade off between these two conflicting criteria. The Pareto optimal set is shown in Figure 5. The compromise solution obtained for scenario 1 is near the kink where the curve flattens in the Pareto plot. Intuitively, this is a good location that provides reasonable balance between the conflicting objectives.

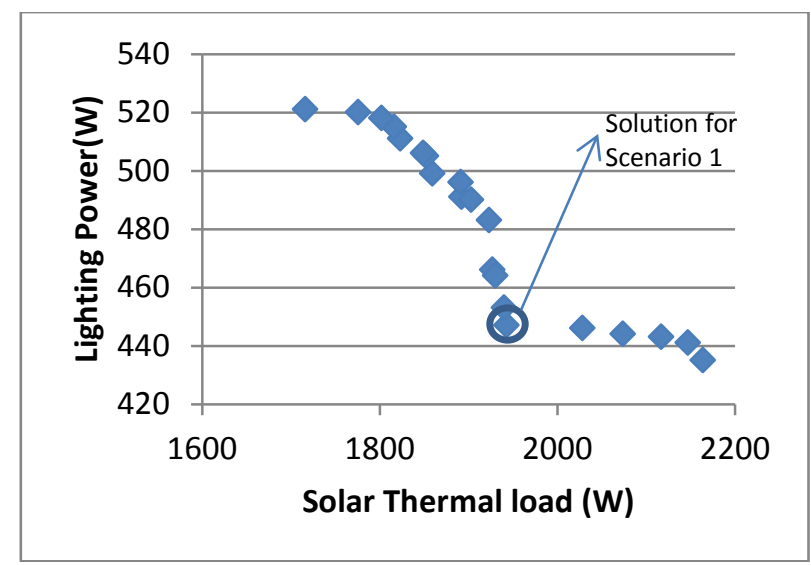

Figure 5. Pareto set for 3 pm 


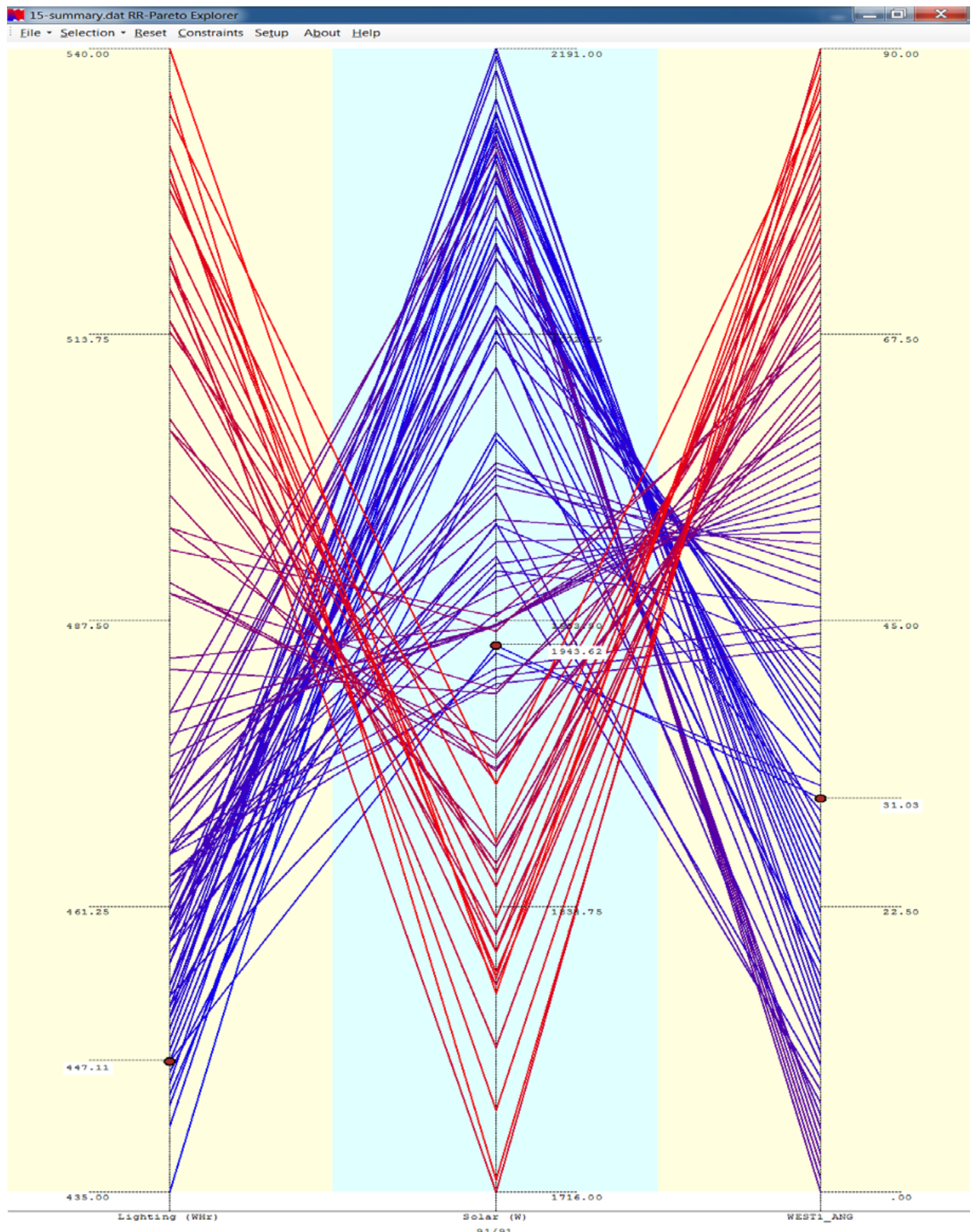

Figure 6. Parallel axis plot showing the trade-off between lighting power and solar thermal load 
Trade-offs between lighting and thermal load are easily visualized using the parallel axis plot shown in Figure 6. In a parallel axis plot, multiple parameters are displayed using vertical axes. In Figure 6, the first axis represents the lighting power, the second axis the solar thermal load and the third axis the optimization variable, the angle of the light shelf. The range of values of these variables are seen in the plot. Each series of lines connecting the axes represent one solution point. For example, the lines connecting the solid dots represent a point close to the compromise solution obtained in Scenario 1. They connect the points lighting power $=$ $447 \mathrm{~W}$, Solar thermal load $=1799 \mathrm{~W}$ and angle $=31$ degrees. The many lines with positive slope starting from low values of lighting power and meeting high values of solar thermal load indicate that these two criteria conflict with each other. That is, lighting power can only be improved at the expense of solar thermal load. Similarly, the lines with negative slope indicate that the solar thermal load can only be reduced by increasing lighting power. The results for simulations at $10 \mathrm{am}$ are presented in Figures 7 and 8.

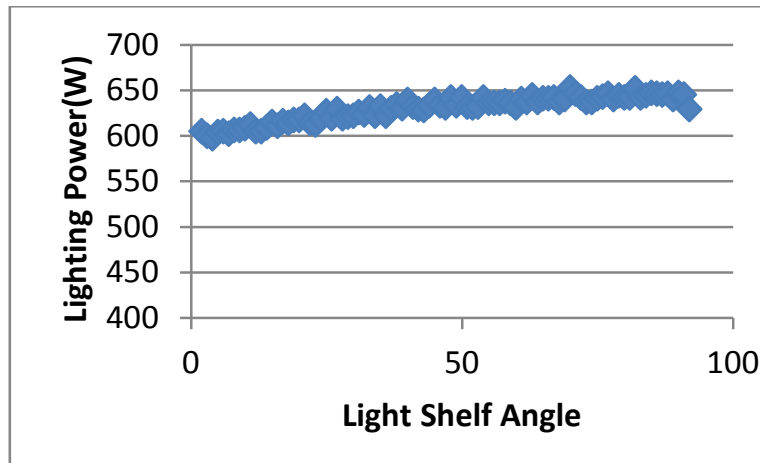

Figure 7. Variation of lighting power with light shelf angle at $10 \mathrm{am}$

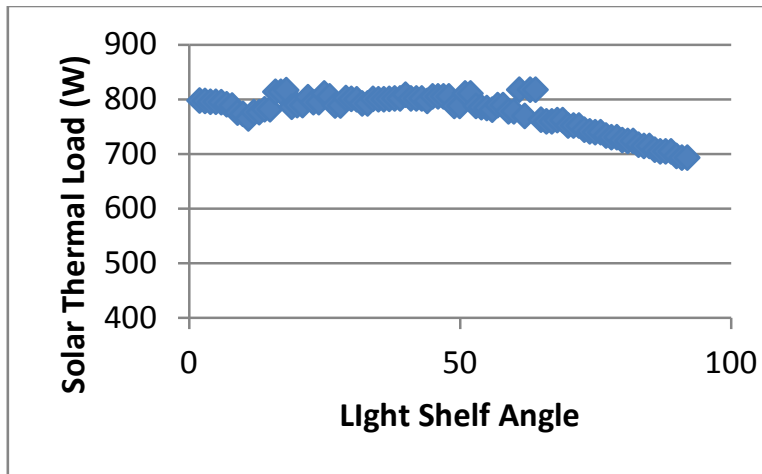

Figure 8. Variation of Solar Thermal Load with light shelf angle at $10 \mathrm{am}$
At $10 \mathrm{am}$, the sun is on the east side and there is negligible effect of the light shelf on direct solar radiation. However, energy simulations show some reduction in the solar thermal load especially at high angles. This is mainly due to the effects of diffuse radiation from the sky. In general, closing the light shelf (by increasing the angle) reduces the solar thermal load while increasing the lighting power consumption due to reduced light transmission.

The compromise solutions obtained for 10 am are summarised in Table 3. The compromise solution obtained in Scenario 1 is the light shelf angle of 2 degrees. This corresponds to lighting power of $597 \mathrm{~W}$ and thermal load of $795 \mathrm{~W}$. For scenario 2, the compromise solution has light shelf angle of 90 degrees. In this case, the light shelf blocks thermal radiations coming from outside allowing no daylight through the upper part of the window.

For scenario 3, the compromise solution is the same as in scenario 2. This is because the light shelf is not very effective as a lighting device at this time and closing the shelf completely does not reduce the lighting power by more than $10 \%$. It is more effective as a shading device that blocks diffuse radiations.

Table 3 Compromise solutions at 10 am

\begin{tabular}{cccc}
\hline Scenario & $\begin{array}{c}\text { Angle } \\
\text { (degree) }\end{array}$ & $\begin{array}{c}\text { Lighting } \\
\text { Power } \\
\text { (W) }\end{array}$ & $\begin{array}{c}\text { Solar } \\
\text { Thermal } \\
\text { load } \\
\text { (W) }\end{array}$ \\
\hline 1 & 2 & 597 & 795 \\
2 & 90 & 629 & 693 \\
3 & 90 & 629 & 693
\end{tabular}

From tables 2 and 3, it is seen that the solutions obtained are different according to the time of the day, the priorities of the user, and the acceptable deterioration in the performance parameters.

In order to evaluate the performance of the adaptive light shelf with respect to a static horizontal light shelf, the energy consumption for all the solutions are compared for 10 am and $3 \mathrm{pm}$ in Tables 4 and 5. The energy for the hour is computed by adding the lighting power and the power required for air conditioning assuming a coefficient of performance (COP) of 6 . At 3 $\mathrm{pm}$ when the sun is on the west, up to $7.6 \%$ savings in total energy is obtained compared to a static light shelf. Even in the morning when the sun is on the east, the light shelf is able to save $1.2 \%$ of the total energy in scenario 1. At $10 \mathrm{am}$ for the scenario 2, there is an increase in the total energy because energy was not 
explicitly used as an optimisation objective. In this case, solar thermal load was the primary objective and the reduction in cooling load actually caused the total energy to increase due to the increase in lighting load.

Table4 Energy Savings at $3 \mathrm{pm}$

\begin{tabular}{cccc}
\hline Scenario & $\begin{array}{c}\text { Angle } \\
\text { (degree) }\end{array}$ & $\begin{array}{c}\text { Energy } \\
\text { (WH) }\end{array}$ & Savings \\
\hline 1 & 31 & 845 & $7.6 \%$ \\
2 & 90 & 894 & $2.3 \%$ \\
3 & 44 & 863 & $5.6 \%$
\end{tabular}

Table 5 Energy Savings at 10 am

\begin{tabular}{cccc}
\hline Scenario & $\begin{array}{c}\text { Angle } \\
\text { (degree) }\end{array}$ & $\begin{array}{c}\text { Energy } \\
\text { (WH) }\end{array}$ & Savings \\
\hline 1 & 2 & 829 & $1.2 \%$ \\
2 & 90 & 894 & $-1.3 \%$ \\
3 & 90 & 863 & $-1.3 \%$
\end{tabular}

\section{$4 \quad$ Future work}

A full-scale laboratory prototype has been constructed to evaluate the actual performance of the adaptive light shelf. The control actions identified by the RRPARETO3 algorithm will be applied to the light shelf. Measurements will be taken to evaluate the lighting and thermal performance under different sky conditions and position of the sun.

\section{Conclusions}

The concept of an adaptive light shelf looks promising. Results from simulations show that significant energy savings can be achieved in comparison to a static light shelf. The multi-objective optimization algorithm RRPARETO3 is able to identify good compromise solutions that make reasonable tradeoffs between two conflicting criteria namely, lighting power consumption and solar thermal load

\section{References}

[1] Radford A.D. and Gero J.S. Design by optimization in architecture, building and construction, New York: Van Nostrand Reinhold; 1987

[2] Horn J. Multicriteria decision making and evolutionary computation. In Handbook of evolutionary computation, Back T, Fogel DB, Michalewicz Z, editors, Bristol, UK: Institute of Physics Publishing., 1997.

[3] Grierson D. E. Pareto multi-criteria decision making, Advanced Engineering Informatics, Volume 22, Issue 3, pp. 371-384, 2008.

[4] Caldas L. Generation of energy-efficient architecture solutions applying GENE_ARCH: An evolution-based generative design system, Advanced Engineering Informatics, Volume 22, Issue 1, January 2008, pp. 59-70, 2008,

[5] Raphael B. Multi-criteria decision making for collaborative design optimization of buildings, Built Environment Project and Asset Management, Vol 1, Issue 2, Nov, 2011.

[6] Aghemo C., Pellegrino A., LoVerso V.R.M. The approach to daylighting by scale models and sun and sky simulators: A case study for different shading systems, Building and Environment, 43 pp. 917-927, 2008.

[7] Raphael, B. Active Control of Daylighting Features in Buildings. Computer-Aided Civil And Infrastructure Engineering, 26(5), pp. 393-405, 2011.

[8] Ward L. G. and Shakespeare R. Rendering with Radiance: the art and science of lighting visualisation. San Francisco: Morgan Kaufmann; 1998.

[9] EnergyPlus, EnergyPlus documentation, http://apps1.eere.energy.gov/buildings/energyplus/ energyplus documentation.cfm, accessed 01/05/2014 